\title{
Asymptotic analysis of some non-linear soft thin layers
}

\author{
F. Lebon ${ }^{\mathrm{a}, *}$, R. Rizzoni ${ }^{\mathrm{b}}, \mathrm{S}$. Ronel-Idrissi ${ }^{\mathrm{c}}$ \\ a Laboratoire de Mécanique et d'Acoustique and Université Aix-Marseille 1, 31, Ch. Joseph Aiguier, 13402 Marseille Cedex 20, France \\ b Dipartimento di Ingegneria, Università di Ferrara, Via Saragat 1, 44100 Ferrara, Italy \\ ${ }^{c}$ Laboratoire Mécanique Matériaux Structures, Université Claude Bernard Lyon 1, 82, Bd Niels Bohr, 69622 Villeurbanne Cedex, France
}

This paper shows how to model thin layers and interfaces by asymptotic techniques. Some behaviors are treated: visco-elasticity (Maxwell, Kelvin-Voigt, Norton), Mohr-Coulomb non-associated elasto-plasticity and non-monotone relationship in the strain-stress diagram. Results are given concerning two kinds of stratified thin layers. Numerical validations and algorithms are proposed and presented.

Keywords: Interface laws; Asymptotic study; Elasto-plasticity; Non-monotone behavior; Finite elements

\section{Introduction}

Contact and interface mechanics intervenes more and more often in computational structures. To have reliable tools to size more and more complex systems, it is indispensable to take into account in a precise way the connections between the various solids of the structure. This consideration can be very complex because of the strongly non-linear character and possibly imperfect and very localized of these connections. For examples, in the modelling of the processes of metal forming, consideration of the friction is necessary because it pilots the whole process; the modelling of the mortar in the case of masonry structures is necessary to estimate in a precise way the mechanical characteristics of the structures, their risks of ruin and collapse, etc.

The purpose of this paper is to show how it is possible to obtain families of interface laws from the mechan-

\footnotetext{
* Corresponding author.
}

ical behavior of thin layers. The consideration of bonded joints in real structures can lead from a numerical point of view to problems of too large sizes, especially if these joints have a non-linear behavior $[1,3]$. From a general way, one is going to consider joints of weak thickness and weak rigidity with regard to those of the substrata. One has then to deal with problems taking into account at least two small parameters (the thickness, the rigidity). An asymptotic study (a micro-macro passage), completed by numerical calculations, leads to so-called "asymptotic contact laws" who allow describing the (macroscopic) mechanical behavior of the interfaces (see references in $[1,3]$ ).

Section 2 is devoted to the mechanical problem. In the third section the mathematical background is recalled. We consider in this paper various kinds of nonlinear behavior for the thin layers:

- visco-elasticity (Maxwell, Kelvin-Voigt or Norton type): one explicits the results expressed in [2] (Section 4); 
- non-associated elasto-plastic of Mohr-Coulomb type (Section 5);

- non-monotone relationship in the strain-stress diagram (Section 6).

One can show in the first and second cases, that the interface laws obtained are of Tresca or Coulomb kind The last case can model instabilities on the contact boundary. In Section 7, the thin layer is supposed to be a stratified composite. This case permits to show how it is possible to add various behavior laws. We obtain the same kind of results that proposed in [4]. The last section is devoted to numerical algorithms.

\section{The mechanical problem}

The aim of this section is to present the mechanical problem and to give the notations used in the paper. We consider a body occupying an open bounded set $\Omega$ of $R^{3}$, with smooth boundary $\partial \Omega$, the three dimensional space is referred to the orthonormal frame $\left(O, x_{1}, x_{2}, x_{3}\right)$. This body is supposed to have a non-empty intersection $S$ with the plane $\left\{x_{3}=0\right\}$. Let $\varepsilon>0$, a parameter intended to tend to zero, we denote

$B^{\varepsilon}=\left\{x=\left(x_{1}, x_{2}, x_{3}\right) \in \Omega \quad\right.$ such that $\left.\left|x_{3}\right|<\frac{\varepsilon}{2}\right\}$,

$\Omega^{\varepsilon}=\left\{x=\left(x_{1}, x_{2}, x_{3}\right) \in \Omega \quad\right.$ such that $\left.\left|x_{3}\right|>\frac{\varepsilon}{2}\right\}$,

$\Omega_{ \pm}^{\varepsilon}=\left\{x=\left(x_{1}, x_{2}, x_{3}\right) \in \Omega \quad\right.$ such that $\left.\pm x_{3}>\frac{\varepsilon}{2}\right\}$,

$S_{ \pm}^{\varepsilon}=\left\{x=\left(x_{1}, x_{2}, x_{3}\right) \in \Omega \quad\right.$ such that $\left.\pm x_{3}=\frac{\varepsilon}{2}\right\}$,

$\Omega_{ \pm}=\left\{x=\left(x_{1}, x_{2}, x_{3}\right) \in \Omega \quad\right.$ such that $\left.\pm x_{3}>0\right\}$,

$S=\left\{x=\left(x_{1}, x_{2}, x_{3}\right) \in \Omega \quad\right.$ such that $\left.x_{3}=0\right\}$,

$\Omega_{0}=\Omega_{+} \cup \Omega_{-}$.

In the following, we suppose that $B^{\varepsilon}$ is the domain occupied by the joint and $\Omega^{\varepsilon}$ by the deformable bodies (see Fig. 1), $\Omega^{0}$ being the geometrical limit of $\Omega^{\varepsilon} . S^{\varepsilon}$ is the

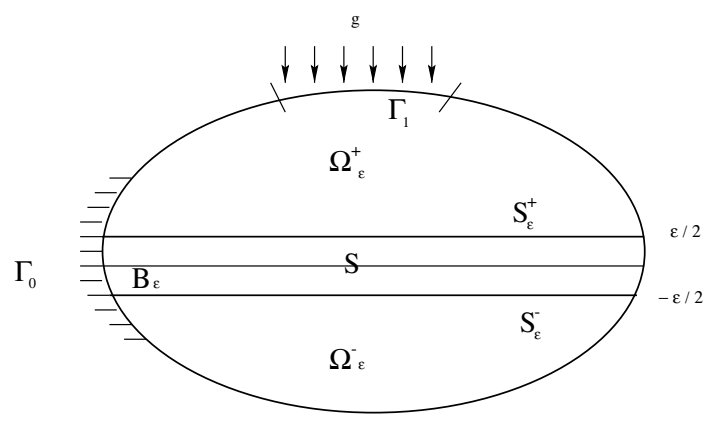

Fig. 1. Geometry of the problem. interface between the adhesive and the adherents, and $S$ is the surface to which the adhesive tends geometrically. On the structure is applied a body force density $\varphi$ and a surface force density $g$ on $\Gamma_{1}$ a part of the boundary, $n$ denotes the external unit normal vector to $\Omega$. The part $\Gamma_{0}$ of the boundary is supposed to be embedded. The two bodies are supposed to be elastic and the joint will be considered successively as elastic, visco-elastic, elasto-plastic and pseudo-elastic. We denote by $\sigma^{\varepsilon}$ the stress tensor and $u^{\varepsilon}$ the displacement field. Under the small perturbations hypothesis, the strain tensor is written

$e_{i j}\left(u^{\varepsilon}\right)=\frac{1}{2}\left(\frac{\partial u_{i}^{\varepsilon}}{\partial x_{j}}+\frac{\partial u_{j}^{\varepsilon}}{\partial x_{i}}\right)$.

If $\psi$ is a function, we define by $\psi\left(x_{0}^{+}\right)$(resp. $\psi\left(x_{0}^{-}\right)$) the limit of $\psi(x)$ when $x$ tends to $x_{0}, x \geqslant x_{0}$ (resp. $x \leqslant x_{0}$ ). In order to define the interface laws between bodies and the joint, we have to introduce some notations. We define the jump of a function on the interfaces

$$
\begin{aligned}
& {[v]_{ \pm}=v\left(x_{1}, \pm \frac{\varepsilon^{+}}{2}\right)-v\left(x_{n 1} \pm \frac{\varepsilon^{-}}{2}\right),} \\
& {[v]=v\left(x_{1}, 0^{+}\right)+v\left(x_{1}, 0^{-}\right) .}
\end{aligned}
$$

In the following sections, the adhesion between the bodies and the joint will be supposed to be perfect, that is

$\left[u^{\varepsilon}\right]_{ \pm}=0 \quad$ on $S_{ \pm}^{\varepsilon}$.

We denote by $a_{i j k l}$ the elasticity parameters of the adherents. Thus, we have to solve the following problem

$$
\left(P_{\varepsilon}\right) \begin{cases}\text { Find }\left(u^{\varepsilon}, \sigma^{\varepsilon}\right) \text { such that : } & \\ \sigma_{i j, j}^{\varepsilon}=-\varphi_{i} & \text { in } \Omega, \\ \sigma_{i j}^{\varepsilon}=a_{i j k h} e_{k h}\left(u^{\varepsilon}\right) & \text { in } \Omega^{\varepsilon}, \\ + \text { behavior laws } & \text { in } B^{\varepsilon}, \\ u^{\varepsilon}=0 & \text { on } \Gamma_{0}, \\ \sigma^{\varepsilon} n=g & \text { on } \Gamma_{1}, \\ {\left[u^{\varepsilon}\right]_{ \pm}=\left[\sigma^{\varepsilon}\right]_{ \pm}=0} & \text { on } S_{ \pm}^{\varepsilon} .\end{cases}
$$

By convention, we define the local frame $\left(n^{ \pm}, t^{ \pm}\right)$where $n^{ \pm}$is the external normal vector to $\Omega_{ \pm}^{\varepsilon}$. In the same way, we define $(n, t)$, where $n$ is external normal to $\Omega_{+}$. In these local basis, a vector $v$ will decomposed in normal and tangential part as follow:

$$
\begin{aligned}
& v\left(x_{1}, \pm \frac{\varepsilon^{+}}{2}\right)=v_{N}\left(x_{1}, \pm \frac{\varepsilon^{+}}{2}\right) n^{ \pm}+v_{T}\left(x_{1}, \pm \frac{\varepsilon^{-}}{2}\right), \\
& v\left(x_{1}, 0^{ \pm}\right)=v_{N} n+v_{T} .
\end{aligned}
$$

\section{Mathematical background}

The idea of matched asymptotic expansions [5] (other techniques are given in [2-4,6-8]) is to find two expansions of the displacement $u^{\varepsilon}$ and to the stress $\sigma^{\varepsilon}$ in pow- 
ers of $\varepsilon$, that is, an external one in the bodies and an internal in the joint, and to connect these two expansions in order to obtain the same limit. In the following, we have considered a problem in dimension 2 in order to simplify the computations. The external expansion is a classical expansion in powers of $\varepsilon$

$$
\begin{aligned}
& u^{\varepsilon}\left(x_{1}, x_{2}\right)=u^{0}\left(x_{1}, x_{2}\right)+\varepsilon u^{1}\left(x_{1}, x_{2}\right)+\cdots, \\
& \sigma_{i j}^{\varepsilon}\left(x_{1}, x_{2}\right)=\sigma_{i j}^{0}\left(x_{1}, x_{2}\right)+\varepsilon \sigma_{i j}^{1}\left(x_{1}, x_{2}\right)+\cdots, \\
& e_{i j}\left(u^{\varepsilon}\right)\left(x_{1}, x_{2}\right)=e_{i j}^{0}+\varepsilon e_{i j}^{1}+\cdots, \\
& \left.e_{i j}^{l}=\frac{1}{2} \frac{\partial u_{i}^{l}}{\partial x_{j}}+\frac{\partial u_{j}^{l}}{\partial x_{i}}\right)
\end{aligned}
$$

In the internal expansion, we proceed to a blow-up of the second variable. Let $y_{2}=\frac{x_{2}}{\varepsilon}$. The internal expansion gives

$$
\begin{aligned}
& u^{\varepsilon}\left(x_{1}, x_{2}\right)=v^{0}\left(x_{1}, y_{2}\right)+\varepsilon v^{1}\left(x_{1}, y_{2}\right)+\cdots, \\
& \sigma_{i j}^{\varepsilon}\left(x_{1}, y_{2}\right)=\varepsilon^{-1} \tau_{i j}^{-1}\left(x_{1}, y_{2}\right)+\tau_{i j}^{0}\left(x_{1}, y_{2}\right)+\varepsilon \tau_{i j}^{1}\left(x_{1}, y_{2}\right)+\cdots, \\
& e_{i j}\left(u^{\varepsilon}\right)\left(x_{1}, y_{2}\right)=\varepsilon^{-1} e_{i j}^{-1}+e_{i j}^{0}+\varepsilon e_{i j}^{1}+\cdots, \\
& e_{11}^{l}=\frac{\partial v_{1}^{l}}{\partial x_{1}}, \quad e_{22}^{l}=\frac{\partial v_{2}^{l+1}}{\partial y_{2}}, \quad e_{12}^{l}=\frac{1}{2}\left(\frac{\partial v_{2}^{l}}{\partial x_{1}}+\frac{\partial v_{1}^{l+1}}{\partial y_{2}}\right)
\end{aligned}
$$

The third step of the method consists in the matching of the two expansions. The idea is to define some intermediate points where the expansions can be superposed. In particular, we observe that when $\varepsilon$ tends to zero, $x_{2}$ tends to $0^{ \pm}$and $y_{2}$ tends to $\pm \infty$. The matching of the two expansions gives

$$
\begin{aligned}
& v^{0}\left(x_{1}, \pm \infty\right)=u^{0}\left(x_{1}, 0^{ \pm}\right), \\
& \tau^{-1}\left(x_{1}, \pm \infty\right)=0, \quad \tau^{0}\left(x_{1}, \pm \infty\right)=\sigma^{0}\left(x_{1}, 0^{ \pm}\right) .
\end{aligned}
$$

We observe that in the equilibrium equations appear terms of order -2 and -1 . The equilibrium equation at order -2 gives

$\frac{\partial \tau_{i 2}^{-1}}{\partial y_{2}}=0$.

Thus, $\tau_{i 2}^{-1}$ does not depend on $y_{2}$. Due to the limit of $\tau_{i 2}^{-1}$ in $\pm \infty$, that is zero, we have

$\tau_{i 2}^{-1}=0$.

The equilibrium equation at order -1 gives

$\frac{\partial \tau_{i 2}^{0}}{\partial y_{2}}=0$.

Thus,

$\tau_{i 2}^{0}=\sigma_{i 2}^{0}\left(x_{1}, 0^{ \pm}\right)$.

Note that in the following, $\bar{f}=\lim _{f, \varepsilon \rightarrow 0} \frac{f}{\varepsilon}$. In particular, we denote by $\bar{\lambda}$ and $\bar{\mu}$ the limits associated to the Lamé's coefficients.

\section{Visco-elasticity}

\subsection{Kelvin-Voigt visco-elasticity}

We consider that the thin layer is visco-elastic and obeys to the Kelvin-Voigt's law of linear visco-elasticity. We recall that we consider a perfect adhesion between the bodies and the glue. The deformation tensor is splitted in two parts: an elastic part and a non-elastic one. The symbol (') characterizes the time derivative. The behavior law in the thin layer is given by

$\sigma_{i j}^{\varepsilon}=\lambda\left(e_{k k}+\theta_{\lambda} \dot{e}_{k k}\right) \delta_{i j}+2 \mu\left(e_{i j}+\theta_{\mu} \dot{e}_{i j}\right)$,

where $\lambda$ and $\mu$ are the Lamé's coefficients. $\theta_{\lambda}$ and $\theta_{\mu}$ are two characteristic times [9].

The asymptotic expansion gives

$$
\begin{aligned}
\varepsilon^{-1} \tau_{i j}^{-1} & +\tau_{i j}^{0}+\varepsilon^{1} \tau_{i j}^{1}+\cdots \\
= & \lambda\left(\varepsilon^{-1}\left(e_{k k}^{-1}+\theta_{\lambda} \dot{e}_{k k}^{-1}\right)+e_{k k}^{0}+\theta_{\lambda} \dot{e}_{k k}^{0}\right. \\
& \left.+\varepsilon\left(e_{k k}^{1}+\theta_{\lambda} \dot{e}_{k k}^{1}\right)+\cdots\right) \delta_{i j}+2 \mu\left(\varepsilon^{-1}\left(e_{i j}^{-1}+\theta_{\mu} \dot{e}_{i j}^{-1}\right)\right. \\
& \left.+e_{i j}^{0}+\theta_{\mu} \dot{e}_{i j}^{0}+\varepsilon\left(e_{i j}^{1}+\theta_{\mu} \dot{e}_{i j}^{1}\right)+\cdots\right) .
\end{aligned}
$$

In the internal expansion, we observe that the important quantities are the ratio between the Lamé's coefficients (resp. the characteristic times) and the thickness of the thin layer. In order to simplify, we suppose that the characteristic times are constant values regarding to the thickness. The other cases can be deduced easily by the techniques presented in the following. We obtain using the notation defined in Section 3

$\tau_{i j}^{-1}=0, \quad \tau_{i j}^{0}=\bar{\lambda}\left(e_{k k}^{-1}+\theta_{\lambda} \dot{e}_{k k}^{-1}\right) \delta_{i j}+2 \bar{\mu}\left(e_{i j}^{-1}+\theta_{\mu} \dot{e}_{i j}^{-1}\right)$.

Thus, by integration in the interval $[-1 / 2,1 / 2]$ and using Eqs. (9), (10) and (14), we obtain

$\begin{aligned} \sigma_{12}^{0} & =\bar{\mu}\left[u_{1}^{0}\right]+\bar{\mu} \theta_{\mu}\left[\dot{u}_{1}^{0}\right], \\ \sigma_{22}^{0} & =(\bar{\lambda}+2 \bar{\mu})\left[u_{2}^{0}\right]+\left(\bar{\lambda} \theta_{\lambda}+2 \bar{\mu} \theta_{\mu}\right)\left[\dot{u}_{2}^{0}\right] .\end{aligned}$

To summarize, the interface law is given by

$\sigma n=K_{L}[u]+K_{\theta}[\dot{u}]$.

The diagonal matrices $K_{L}$ and $K_{\theta}$ are deduced easily from (16).

We observe that this interface law is valid for a large number of coefficients variations. Particularly, if the limits of the ratios are infinite, the jumps in the former formulas are equal to zero (perfect adhesion). Conversely, if the coefficients are equal to zero, the stress tensor is equal to zero (perfect sliding).

\subsection{Maxwell visco-elasticity}

The thin layer is supposed to obey to the Maxwell's law of visco-elasticity. The behavior law in the thin layer is given by [9] 
$e\left(u^{\varepsilon}\right)=\frac{1+v}{E}\left(\dot{\sigma}_{i j}^{\varepsilon}+\frac{\sigma_{i j}^{\varepsilon}}{t_{1}}\right)-\frac{v}{E}\left(\dot{\sigma}_{k k}^{\varepsilon}+\frac{\sigma_{k k}^{\varepsilon}}{t_{2}}\right) \delta_{i j}$.

$E$ is the Young's modulus and $v$ is the Poisson's ratio. $t_{1}$ and $t_{2}$ are two characteristic times. We use the same techniques that in the previous section. We assume that $t_{1}=t_{2}$. The important value in the expansion is the ratio between the Young's modulus and the thickness of the thin layer. Using the notation of Section 3, we obtain

$\left[u_{1}^{0}\right]=\frac{1+v}{\bar{E}}\left(\dot{\sigma}_{12}^{0}+\sigma_{12}^{0} / t_{1}\right)$,

$\left[u_{2}^{0}\right]=-\frac{1-v}{\bar{E} v}\left(\dot{\sigma}_{22}^{0}+\sigma_{22}^{0} / t_{1}\right)$.

The interface law is written

$[u]=K_{Y} \dot{\sigma} n+K_{t} \sigma n$.

The matrices $K_{Y}$ and $K_{t}$ are deduced easily from Eq. (19).

\subsection{Norton visco-elasticity}

The thin layer obeys to the non-linear Norton's law of visco-elasticity. The behavior law in the thin layer is given by

$\sigma_{i j}^{\varepsilon}=\lambda e_{k k} \delta_{i j}+2 \mu e_{i j}+\eta|\dot{e}|^{p-2} \dot{e}_{i j}$,

where $\lambda$ and $\mu$ are the Lamé's coefficients and $\eta$ is the viscosity coefficient. $p$ is a scalar parameter. We use the same techniques that in previous sections. The characteristic coefficients in the expansions are the ratios between the Lamé's coefficients and the thickness of the thin layer and the ratio between the viscosity coefficient and the thickness at the power $p-1$. Using the notation of the previous sections, we obtain

$\sigma_{12}^{0}=\bar{\mu}\left[u_{1}^{0}\right]+\bar{\eta} / 2 \int_{-1 / 2}^{1 / 2}|\dot{e}|^{p-2} \frac{\partial \dot{v}_{1}^{0}}{\partial y_{2}} \mathrm{~d} y_{2}$,

$\sigma_{22}^{0}=(\bar{\lambda}+2 \bar{\mu})\left[u_{2}^{0}\right]+\bar{\eta} \int_{-1 / 2}^{1 / 2}|\dot{e}|^{p-2} \frac{\partial \dot{v}_{2}^{0}}{\partial y_{2}} \mathrm{~d} y_{2}$.

We have to solve two differential equations. The stresses $\sigma_{12}^{0}$ and $\sigma_{22}^{0}$ depend only on $x_{1}$, thus $\frac{\partial \dot{v}_{1}^{0}}{\partial y_{2}}$ and $\frac{\partial \dot{v}_{2}^{0}}{\partial y_{2}}$ do not depend on $y_{2}$. The displacements $\dot{v}_{1}^{0}$ and $\dot{v}_{2}^{0}$ depend linearly on $y_{2}$. We deduce by integration

$$
\begin{aligned}
& \sigma_{12}^{0}=\bar{\mu}\left[u_{1}^{0}\right]+\bar{\eta} / 2\left|[\dot{u}] \otimes_{s} n\right|^{p-2}\left[\dot{u}_{1}^{0}\right], \\
& \sigma_{22}^{0}=(\bar{\lambda}+2 \bar{\mu})\left[u_{2}^{0}\right]+\bar{\eta}\left|[\dot{u}] \otimes_{s} n\right|^{p-2}\left[\dot{u}_{2}^{0}\right],
\end{aligned}
$$

where the symbol $\otimes_{s}$ denotes the symmetric tensorial product. To summarize the interface law is written

$\sigma n=K_{L}[u]+K_{\eta}\left|[\dot{u}] \otimes_{s} n\right|^{p-2}[\dot{u}]$.

The matrices $K_{L}$ and $K_{\eta}$ are deduced easily from Eq. (23).

\subsection{Incompressibility}

As in the previous sections, we consider a perfect adhesion between the bodies and the glue. The incompressibility condition is written in the quasi-static case

$\operatorname{div} u=0$.

The former equation becomes

$\varepsilon^{-1} \frac{\partial u_{2}}{\partial y_{2}}+\frac{\partial u_{1}}{\partial x_{1}}+\cdots=0$.

Thus,

$\frac{\partial u_{2}}{\partial y_{2}}=0$

and

$\left[u_{2}\right]=0$.

We obtain the law of bilateral contact

$\left[u_{N}\right]=0$.

\section{Mohr-Coulomb elasto-plasticity}

\subsection{Mathematical results}

As in the previous sections, we consider a perfect adhesion between the bodies and the glue. The deformation is decomposed in an elastic part and in a plastic one. The thin layer obeys to the Mohr-Coulomb's law of elasto-plasticity, that is

$$
\begin{aligned}
& e\left(u^{\varepsilon}\right)=e^{e}+e^{p}, \\
& \sigma_{i j}^{\varepsilon}=\lambda e_{k k} \delta_{i j}+2 \mu e_{i j}, \\
& \left|\sigma_{t}\right| \leqslant C-\tan \Omega\left|\sigma_{n}\right|, \\
& I f\left|\sigma_{t}\right|<C-\tan \Omega\left|\sigma_{n}\right| \text { then } \dot{e}^{p}=0, \\
& I f\left|\sigma_{t}\right|<C-\tan \Omega\left|\sigma_{n}\right| \text { then } \dot{e}^{p}=k \bar{n} \otimes_{s} \bar{t},
\end{aligned}
$$

where $\Omega$ is the friction angle, $C$ is the cohesion strength, $\sigma_{n}$ and $\sigma_{t}$ are respectively the normal and the tangential stresses; we denote by $\bar{n}$ and $\bar{t}$ the normal and tangential directions of the face corresponding to the plastic yield, $k$ is a non-negative parameter.

We proceed as in the former sections. We consider the same case than in Section 3. The internal expansion gives using the same techniques that in the previous paragraphs

$\tau_{12}^{0}=\bar{\mu}\left(\frac{\partial u_{1}}{\partial y_{2}}-e_{12}^{p}\right), \quad \tau_{22}^{0}=(\bar{\lambda}+2 \bar{\mu})\left(\frac{\partial u_{2}}{\partial y_{2}}-e_{22}^{p}\right)$.

Before the beginning of the plastification, by integration and using the connection conditions, we find the elastostatic case.

$\sigma \bar{n}=K_{L}[u]$. 
The most interesting case is when the plastic yield is reached, we obtain in this case by integration

$\tau_{12}^{0}=\bar{\mu}\left(\left[u_{1}^{0}\right]-\left[u_{1}^{p}\right]\right), \quad \tau_{22}^{0}=(\bar{\lambda}+2 \bar{\mu})\left(\left[u_{2}^{0}\right]-\left[u_{2}^{p}\right]\right)$.

In other terms and using the connection conditions

$\sigma \bar{n}=K_{L}\left([u]-\left[u^{p}\right]\right)$.

The plastic flow is given by

$\left[\dot{u}^{p}\right]=k \bar{t}$

with $\left[\dot{u}_{i}^{p}\right]=\int_{-1 / 2}^{1 / 2} \dot{e}_{i 2}^{-1, p} \mathrm{~d} y_{2}$.

The limit problem is quite different in this non-linear case. The plastic yield and the plastic flow depend on a local problem because they do not depend on the stress vector but on all the components of the stress tensor. In order to determine the threshold and the direction of sliding, we have to solve the following elasto-plastic problem in the rectangle domain $S \times[-1 / 2,1 / 2]$

$\frac{\partial \tau_{i 2}^{0}}{\partial y_{2}}=0$,

$e^{-1}=e^{e}+e^{p}$,

$\tau_{i j}^{0}=\lambda e_{k k}^{-1} \delta_{i j}+2 \mu e_{i j}^{-1}$,

$\left|\tau_{t}^{0}\right| \leqslant C-\tan \Omega\left|\tau_{n}^{0}\right|$

If $\left|\tau_{t}^{0}\right|<C-\tan \Omega\left|\tau_{n}^{0}\right|$ then $\dot{e}^{p}=0$

If $\left|\tau_{t}^{0}\right|<C-\tan \Omega\left|\tau_{n}^{0}\right|$ then $\dot{e}^{p}=k \bar{n} \otimes_{s} \bar{t}$.

Due to the fact that the solution does not depend on the thickness, we have to solve only a "one-dimensional problem".

We observe that we obtain a "pseudo-penalized-Coulomb" law of friction. If the direction of flow is equal to (or approximated by) $x_{1}$, we find the classical Coulomb law of friction.

\subsection{Numerical validations}

The numerical example presented in this section is the problem of an elastic body bonded to a rigid body by an elasto-plastic thin layer (Fig. 2). The ratio thickness of the thin layer-dimension of the body is equal to 1/ 100. Numerical results are shown in Fig. 3. We observe that the theoretical law is verified with a good agreement. The right part of the figure (elastic behavior) shows that the ratio between the tangential force and the tangential displacement, computed on the boundary elastic body/thin layer, is equal to the coefficient $\bar{\mu}$. In the left part of Fig. 3, we observe the plastification of the thin layer corresponding to a non-linear sliding.

\subsection{Remarks on a simplified behavior}

In this section, we consider a material which obeys to a mono-directional law of Mohr-Coulomb. That is the direction of flow is known. In order to simplify, we consider that the direction is $x_{1}$. The flow rule in Section 5.1 is replaced by

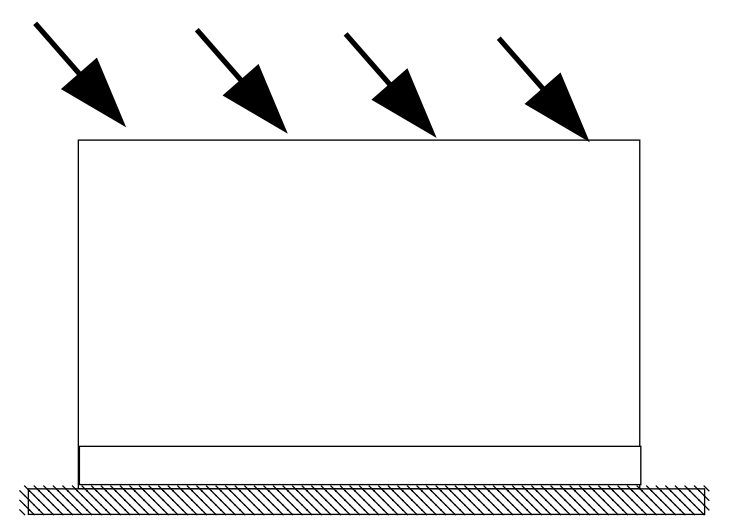

Fig. 2. A long steel bar $(100 \mathrm{~mm} \times 100 \mathrm{~mm})$ bonded with a rigid obstacle. Adhesive: $\varepsilon=1 \mathrm{~mm}, C=0, \Omega=30, E=30 \mathrm{GPa}, v=$ 0.3 ; adherent: $E=210 \mathrm{GPa}, v=0.3$; loadings: $(0.164,-0.0164)$ $\mathrm{N} / \mathrm{mm}$; finite elements 8 -nodes squares.

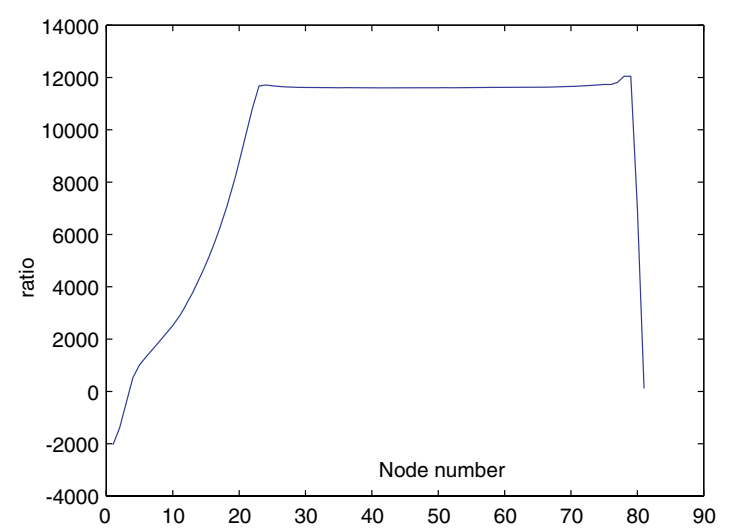

Fig. 3. Ratio tangential force-tangential displacement on the contact zone: beginning of the plastification in the left part of the boundary.

$\dot{e}^{p}=k x_{1} \quad$ and $\quad(\bar{n}, \bar{t})=\left(-x_{2}, x_{1}\right)$.

The asymptotic expansions used in the previous sections give

$\sigma n=K_{L}\left([u]-\left[u^{p}\right]\right)$,
$\left[\dot{u}^{p}\right]=k x_{1}$

In this case, it is not necessary to compute the local problem presented in Section 5.1.

\section{Some remarks on non-monotone behavior}

\subsection{Mathematical results and limitations}

We consider a perfect adhesion between the bodies and the glue. The behavior of the material is non-monotone, that is [15] 
$\sigma_{i j}=\lambda e_{k k} \delta_{i j}+2 \mu e_{i j}+\alpha(T) \chi_{i j}$ if $\alpha(T) \chi_{i j} e_{i j} \leqslant-C(T)$,

$\sigma_{i j}=\lambda e_{k k} \delta_{i j}+2 \mu e_{i j} \quad$ if $\left|\alpha(T) \chi_{i j} e_{i j}\right| \leqslant C(T)$,

$\sigma_{i j}=\lambda e_{k k} \delta_{i j}+2 \mu e_{i j}-\alpha(T) \chi_{i j}$ if $\alpha(T) \chi_{i j} e_{i j} \geqslant C(T)$,

where $T$ is the temperature, $C(T)$ and $\alpha(T)$ are the nonnegative parameters of the law, and $\chi$ is a given orientation tensor. Using the same techniques and the same notations than in the previous sections, the internal expansion, in the case

$\alpha(T) \chi_{i j} e_{i j} \geqslant C(T)$

leads to

$\tau_{12}^{0}=\bar{\mu} \frac{\partial u_{1}^{0}}{\partial y_{2}}-\bar{\alpha} \chi_{12}, \quad \tau_{22}^{0}=(\bar{\lambda}+\bar{\mu}) \frac{\partial u_{2}^{0}}{\partial y_{2}}-\bar{\alpha} \chi_{22}$,

where $\bar{\alpha}$ is the limit of the ratio between the coefficient $\alpha(T)$ and the thickness at the power $\eta<1$. In the following, $\bar{C}$ will denote the ratio between $C(T)$ and the same power of the thickness. By integration, we obtain

$\sigma_{12}^{0}=\bar{\mu}\left[u_{1}^{0}\right]-\bar{\alpha} \chi_{12}, \quad \sigma_{22}^{0}=(\bar{\lambda}+\bar{\mu})\left[u_{1}^{0}\right]-\bar{\alpha} \chi_{22}$.

Thus, we obtain an interface law written

If $\bar{\alpha} \chi_{i 2}\left[u_{i}\right] \geqslant \bar{C}$ then $\sigma n=K_{L}[u]-\bar{\alpha} \chi n$.

An interesting case is $\chi_{22}=0$. Thus, we obtain a normal compliance law and a non-monotone law of friction $[11,12]$.

\subsection{A $1 D$ example}

The previous computations are not totally true because the problem does not have an unique solution. In fact, in the asymptotic expansions particular solutions are chosen. In the previous computations there is no notion of stability which is now classical for this kind of non-monotone laws (see [13] and references therein). In this paragraph we treat the example of a bar of length $l$ occupying the interval $] 0, l[$. In the interval $] 0, \varepsilon l[$, the material has a behavior as in the previous section with a rigidity $k$; in the interval ] $\varepsilon l, l[$, the material is elastic with a rigidity $K$. We suppose that a given displacement $\delta>0$ is applied at the extremity $x=l$. The bar is embedded at the extremity $x=0$. The adhesion is perfect for $x=\varepsilon l$. We denote $u_{a}$ the displacement field in $] 0, \varepsilon l[$ and $u_{b}$ the displacement field in the interval $] \varepsilon l, l[$. We introduce $W_{\min }$ the stored energy of the two-phase part and $W$ the stored energy of the elastic part. The total energy of the composite bar is given by

$E\left(u_{a}, u_{b}\right)=\int_{0}^{\varepsilon l} W_{\min }\left(u_{a, x}(x)\right) \mathrm{d} x+\int_{\varepsilon l}^{l} W\left(u_{b, x}(x)\right) \mathrm{d} x$,

$W_{\min }\left(u_{a, x}(x)\right)=\min \left(\frac{k}{2} u_{a, x}^{2}(x), \frac{k}{2} u_{a, x}^{2}(x)-\alpha u_{a, x}(x)+c\right)$,

$W\left(u_{b, x}(x)\right)=\frac{K}{2} u_{b, x}^{2}(x)$.
Note that $W_{\min }$ has two ascending branches, each one corresponding to a different phase. In this paragraph, we look for a particular minimizer of the total energy. We denote $U_{\varepsilon}=u_{a}(\varepsilon l)=u_{b}(\varepsilon l)$. Introducing the convex envelope of $W_{\min }$, denoted $C W_{\min }$, and using Jensen's inequality, we obtain a lower bound of the energy

$$
\begin{aligned}
E\left(u_{a}, u_{b}\right) \geqslant & \int_{0}^{\varepsilon l} C W_{\min }\left(u_{a, x}(x)\right) \mathrm{d} x+\int_{\varepsilon l}^{l} W\left(u_{b, x}(x)\right) \mathrm{d} x \\
\geqslant & \varepsilon l C W_{\min }\left(\frac{1}{\varepsilon l} \int_{0}^{\varepsilon l} u_{a, x}(x) \mathrm{d} x\right) \\
& +l(1-\varepsilon) W\left(\frac{1}{l(1-\varepsilon)} \int_{\varepsilon l}^{l} u_{b, x}(x) \mathrm{d} x\right) \\
\geqslant & \min \left(\varepsilon l C W_{\min }\left(\frac{U_{\varepsilon}}{\varepsilon l}\right)+l(1-\varepsilon) W\left(\frac{\delta-U_{\varepsilon}}{l(1-\varepsilon)}\right)\right) .
\end{aligned}
$$

The lower bound is obtained when

$C W_{\min , u_{a}}\left(\frac{U_{\varepsilon}}{\varepsilon l}\right)=W_{, u_{b}}\left(\frac{\delta-U_{\varepsilon}}{l(1-\varepsilon)}\right)$.

The solutions of the former equation are the intersections of the graph of $\mathrm{CW}_{\min , u_{a}}$, which is a tri-linear function in view of the non-convexity of $W_{\min }$, and the graph of $W_{, u_{b}}$, which is linear (Fig. 4). Three different values of $U_{\varepsilon}$ are obtained corresponding to the three possible intersections of the graphs (see Fig. 4)

- $\delta \leqslant \delta_{0}, U_{\varepsilon}=\frac{K \delta}{K_{\varepsilon}(1-\varepsilon)}$,

- $\delta_{0} \leqslant \delta<\delta_{1}, U_{\varepsilon}=\delta-\frac{\sigma_{m} l(1-\varepsilon)}{K}$,

- $\delta \geqslant \delta_{1}, U_{\varepsilon}=\frac{\alpha l}{K_{\varepsilon}}+\frac{K \delta}{K_{\varepsilon}(1-\varepsilon)}$

with $\quad \sigma_{m}=\frac{k c}{\alpha}-\frac{\alpha}{2}, \quad K_{\varepsilon}^{-1}=\frac{1-\varepsilon}{K}+\frac{\varepsilon}{k}, \quad \delta_{0}=\sigma_{m} l K_{\varepsilon}^{-1} \quad$ and $\delta_{1}=\delta_{0}+\frac{l \varepsilon \alpha}{k}$. Thus, it is possible that a global minimizer of the energy verifies

$u_{a}(x)=\frac{\sigma}{k} x$ if $0 \leqslant x \leqslant \varepsilon S$,

$u_{a}(x)=\frac{\sigma+\alpha}{k}(x-\varepsilon S)$ if $\varepsilon S \leqslant x \leqslant \varepsilon l$,

$u_{b}(x)=\frac{\sigma}{K}(x-l)+\delta \quad$ if $\varepsilon l \leqslant x \leqslant l$,

$\varepsilon S=\varepsilon l\left(1+\frac{\sigma}{\alpha}-\frac{\sigma k}{\alpha K} l-\frac{k}{\alpha} \delta\right)$,

$\sigma=\frac{k}{\varepsilon l} \frac{K}{K_{\varepsilon}} \quad$ if $0 \leqslant \delta \leqslant \delta_{0}$,

$\sigma=\sigma_{m}=k\left(\frac{C}{\alpha}-\frac{\alpha}{2}\right)$ if $\delta_{0} \leqslant \delta \leqslant \delta_{1}$,

$\sigma=\frac{k}{\varepsilon l}\left(\frac{K}{K_{\varepsilon}}+\frac{\alpha l}{K_{\varepsilon}}\right) \quad$ if $\delta>\delta_{1}$.

Note that $\varepsilon S$ denotes the location of the interface between the two phases in the thin layer. To summarize, we obtain three different branches of solution corresponding to the values of the given displacement $\delta$. At this point, it is possible to study the behavior of the dis- 


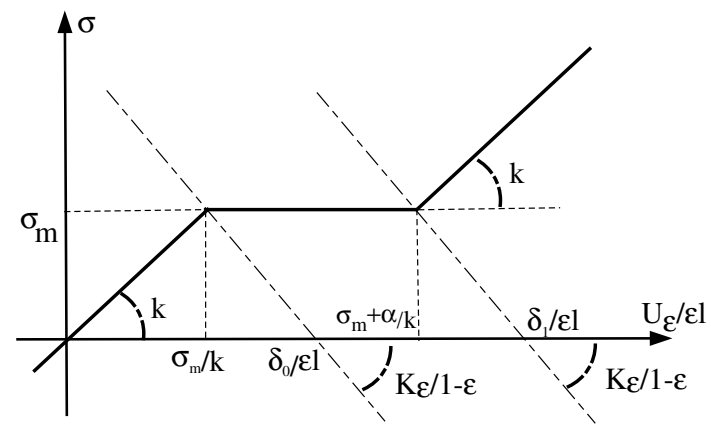

Fig. 4. Solution of the minimization problem.

placement and stress fields when the thickness tends to zero. Thus, using the usual notations, the asymptotic law is given by

$$
\begin{aligned}
& \sigma n=\bar{k}[u] \quad \text { if } 0 \leqslant \delta \leqslant \delta_{0}^{0}, \\
& \sigma n=\frac{l}{K}(\delta-[u]) \quad \text { if } \delta_{0}^{0} \leqslant \delta \leqslant \delta_{1}^{1}, \\
& \sigma n=\bar{k}[u]-\bar{\alpha} \quad \text { if } \delta>\delta_{1}^{0}, \\
& \delta_{0}^{0}=\sigma_{m} l \bar{K}^{-1}, \quad \delta_{1}^{0}=\delta_{0}^{0}+l_{\bar{k}}^{\bar{\alpha}}, \quad \bar{K}^{-1}=\left(\frac{1}{K}+\frac{1}{\bar{k}}\right) .
\end{aligned}
$$

We observe that this law is quite different that the law obtained in Section 6.1. Note that other techniques to determine the asymptotic laws are given in [14].

\section{Stratified thin layers}

\subsection{Introduction}

In this section, it is supposed that the thin layer is a stratified composite with the fiber direction parallel to $x_{1}$ (see Fig. 5). In order to simplify, only two sub-layers of thickness $a \varepsilon$ and $1-a \varepsilon$ are considered $(0 \leqslant a \leqslant 1)$ and a

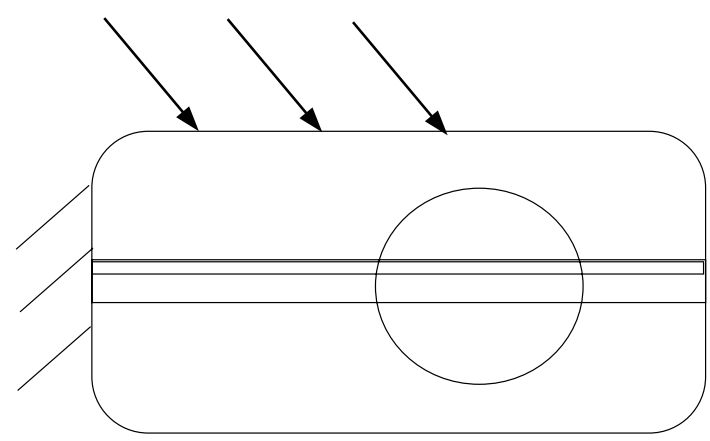

Fig. 5. A stratified bonded joint with an elastic bar $(40 \mathrm{~mm} \times 40$ $\mathrm{mm}$ ). Adhesive: $\varepsilon=0.1 \mathrm{~mm}, a=0.5, \mu_{+}=10 \mathrm{GPa}, \mu_{-}=7.5$ GPa; adherent: $E=130 \mathrm{GPa}, v=0.2$; loadings $(50,-25) \mathrm{MPa}$; finite elements 3-nodes triangles. perfect adhesion between the layers is supposed. We denote

$$
\begin{aligned}
& B_{-}^{\varepsilon}=\left\{x \in B^{\varepsilon} \text { such that }-\frac{\varepsilon}{2}<x_{3} \leqslant \frac{\varepsilon}{2}-a \varepsilon\right\}, \\
& B_{+}^{\varepsilon}=\left\{x \in B^{\varepsilon} \text { such that } \frac{\varepsilon}{2}-a \varepsilon \leqslant x_{3}<\frac{\varepsilon}{2}\right\} .
\end{aligned}
$$

We denote by $\lambda_{-}$and $\mu_{-}$(resp. $\lambda_{+}$and $\mu_{+}$) the Lamé coefficients in $B_{-}^{\varepsilon}$ (resp. $\left.B_{+}^{\varepsilon}\right)$.

\subsection{Elastic layers}

In this paragraph, the two layers are supposed to be elastic. By the same techniques that in the previous sections and using the same usual notations, we obtain

$$
\begin{aligned}
& \sigma_{12}^{0}=\bar{\mu}_{+} \frac{\partial v_{1}^{0}}{\partial y_{2}}, \quad \frac{1}{2}-a \leqslant x_{2}<\frac{1}{2}, \\
& \sigma_{22}^{0}=\left(\bar{\lambda}_{+}+2 \bar{\mu}_{+}\right) \frac{\partial v_{2}^{0}}{\partial y_{2}}, \quad \frac{1}{2}-a \leqslant x_{2}<\frac{1}{2}, \\
& \sigma_{12}^{0}=\bar{\mu}_{-} \frac{\partial v_{1}^{0}}{\partial y_{2}}, \quad-\frac{1}{2}<x_{2} \leqslant \frac{1}{2}-a, \\
& \sigma_{22}^{0}=\left(\bar{\lambda}_{-}+2 \bar{\mu}_{-}\right) \frac{\partial v_{2}^{0}}{\partial y_{2}}, \quad-\frac{1}{2}<x_{2} \leqslant \frac{1}{2}-a .
\end{aligned}
$$

By integration, we obtain

$$
\begin{aligned}
& \sigma_{12}^{0}=\bar{\mu}_{ \pm}\left[u_{1}^{0}\right], \\
& \sigma_{22}^{0}=\left(\bar{\lambda}_{ \pm}+2 \bar{\mu}_{ \pm}\right)\left[u_{2}^{0}\right],
\end{aligned}
$$

where $\bar{\lambda}_{ \pm}$and $\bar{\mu}_{ \pm}$are the geometrical sums of the Lamé coefficients i.e.

$$
\begin{aligned}
& \bar{\lambda}_{ \pm}=\frac{a}{\bar{\lambda}_{+}}+\frac{1-a}{\bar{\lambda}_{-}-}, \\
& \bar{\mu}_{ \pm}=\frac{a}{\bar{\mu}_{+}}+\frac{1-a}{\bar{\mu}_{-}-} .
\end{aligned}
$$

Thus, an elastic interface law is obtain where the coefficients of stiffness are the geometrical sum of the coefficients of stiffness of each thin layer:

$$
\sigma n=K_{L}^{ \pm}[u] \text {. }
$$

The definition of the tensor $K_{L}^{ \pm}$is deduced easily from Eq. 51. An example of validation is given in Fig. 6 on the example of a long bar with two layers with the same thickness.

\subsection{Elastic-plastic layers}

The same kind of study than in the previous section can be done considering that one of these thin layers is perfectly plastic and obeys to the Mohr-Coulomb behavior. In order to simplify, we suppose, as in Section 5.3 , that the behavior of the upper layer is mono-directional. The flow rule in this layer is

$\dot{e}^{p}=k x_{1} \quad$ and $\quad(\bar{n}, \bar{t})=\left(-x_{2}, x_{1}\right)$. 


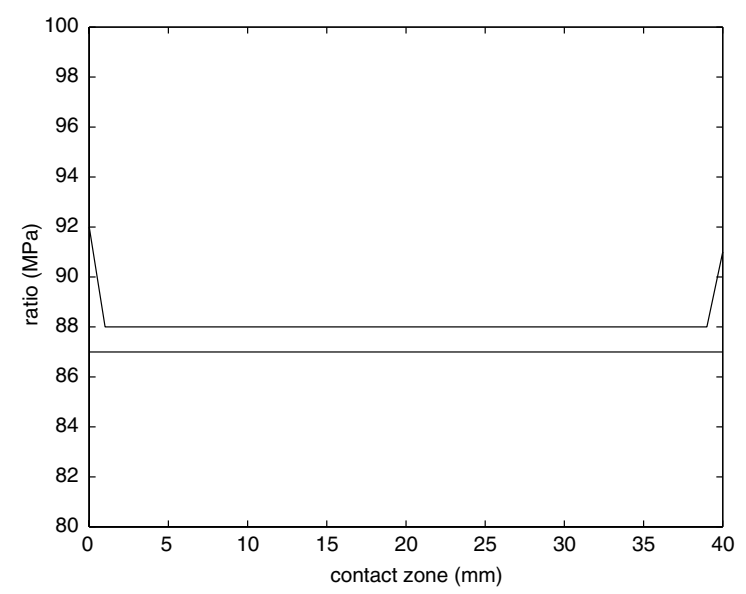

Fig. 6. Ratio tangential force-tangential displacement.

The asymptotic expansions used in the previous sections and the results of the previous section give

$$
\begin{aligned}
& \sigma n=K_{L}^{ \pm}\left([u]-\left[u^{p}\right]\right), \\
& {\left[\dot{u}^{p}\right]=k x_{1} .}
\end{aligned}
$$

We obtain a law which is "classical" in contact mechanics. The next section is devoted to the numerical solution of this kind of problem.

\section{Numerical algorithms}

\subsection{Generalities}

The interface problem introduced in Section 7.3 is written, with $f=\tan \Omega$, Find $\rho$ fixed point of the application $\rho \rightarrow f\left|\sigma_{N}(u(\rho))\right|$, where $u=u(\rho)$ is solution of

$\left\{\begin{array}{l}\text { Find } \mathrm{u} \in \mathrm{V} \text { such that : } \\ J(u) \leqslant J(v)\end{array} \forall v \in V\right.$

with $J(v)=\frac{1}{2} A(v, v)-L(v)+\int_{S} \phi\left(v_{N}\right) \mathrm{d} s+\int_{S} \psi\left(v_{T}\right) \mathrm{d} s, \phi$ is given by

$$
\phi\left(v_{N}\right)= \begin{cases}0 & \text { if } v_{N}<0, \\ \frac{K_{N}}{2}\left(v_{N}\right)^{2} & \text { if } v_{N} \geqslant 0 .\end{cases}
$$

$\psi$ is given by

$$
\psi\left(v_{T}\right)= \begin{cases}-\rho v_{T}-\frac{\rho^{2}}{2 K_{T}} & \text { if } v_{T}<\frac{-\rho}{K_{T}}, \\ \frac{1}{2} K_{T} \cdot\left(v_{T}\right)^{2} & \text { if }\left|v_{T}\right| \leqslant \frac{\rho}{K_{T}}, \\ \rho v_{T}-\frac{\rho^{2}}{2 K_{T}} & \text { if } v_{T}>\frac{\rho}{K_{T}} .\end{cases}
$$

The problem is discretized by a finite element method formulated in displacements. Usually, we use $P 1$ finite elements (triangles with three nodes and six degrees of freedom) or $Q 1$ (quadrangles with four nodes and eight degrees of freedom). We have to minimize a functional still noted $J$ in $R^{2 N P}$. We denote: NP the total number of nodes, NC the number of contact nodes, $I_{\mathrm{N}}$ the indices of normal components of contact nodes, $I_{\mathrm{T}}$ the indices of tangential components of contact nodes, $A$ the stiffness matrix with coefficient $a_{i j}$ and $B$ the stiffness matrix to the surface terms with coefficient $b_{i j}$. The relaxation method consists in finding the solution of the previous problem solving a sequence of minimization problems in $R^{2 N P}$

$$
\left\{\begin{array}{l}
\text { Find } u_{i}^{n+\frac{1}{2}} \text { such that } \forall v \in R^{2 N P} \\
J\left(u_{1}^{n+1}, \ldots, u_{i-1}^{n+1}, u_{i}^{n+\frac{1}{2}}, u_{i+1}^{n}, \ldots, u_{2 N P}^{n}\right) \\
\leqslant J\left(u_{1}^{n+1}, \ldots, u_{i-1}^{n+1}, v, u_{i+1}^{n}, \ldots, u_{2 N P}^{n}\right) .
\end{array}\right.
$$

We denote by $\omega$ the relaxation coefficient. In the first time, we are interested by the normal components.

- $i \in I_{N}$

The algorithm is written

$$
\begin{aligned}
& \left(\begin{array}{l}
u_{i}^{n+\frac{1}{2}}=\frac{1}{d_{i i}^{n+\frac{1}{2}}} \quad \varphi_{i}-\sum_{j=1}^{i-1} d_{i j}^{n+1} u_{j}^{n+1}-\sum_{j=i+1}^{2 N P} d_{i j}^{n} u_{j}^{n} \\
\text { with }
\end{array}\right. \\
& \left\{\begin{array}{l}
d_{i j}^{n}= \begin{cases}a_{i j}+\gamma\left(u_{j}^{n}\right) b_{i j} & \text { if } j \in I_{N}, \\
a_{i j}+\eta\left(u_{j}^{n}\right) b_{i j} & \text { if } j \in I_{T}, \\
a_{i j} & \text { otherwise },\end{cases} \\
\gamma(u)= \begin{cases}0 & \text { if } u \leqslant 0, \\
1 & \text { if } u>0\end{cases}
\end{array}\right. \\
& \text { and } \\
& \begin{array}{l}
\eta(u)= \begin{cases}0 & \text { if }|u|>\frac{\rho}{K_{T}}, \\
1 & \text { if }|u| \leqslant \frac{\rho}{K_{T}},\end{cases} \\
u_{i}^{n+1}=(1-\omega) u_{i}^{n}+\omega u_{i}^{n+\frac{1}{2}} .
\end{array}
\end{aligned}
$$

For tangential components, we have

- $i \in I_{T}$

First, the fixed point problem is considered $\rho_{i}^{l+1}=f\left|\sigma_{N}\left(u\left(\rho_{i}^{l}\right)\right)\right|$ and we write

$$
\left\{\begin{array}{l}
\left.u_{i}^{n+\frac{1}{2}}=\frac{1}{d_{i i}^{n+\frac{1}{2}}} \quad \varphi_{i}-\sum_{j=1}^{i-1} d_{i j}^{n+1} u_{j}^{n+1}-\sum_{j=i+1}^{2 N P} d_{i j}^{n} u_{j}^{n}-\theta\left(u_{i}^{n+\frac{1}{2}}\right) \cdot \rho_{i}^{l}\right) \\
\text { with } \begin{array}{ll}
\theta(u)= \begin{cases}-1 & \text { if } u<\frac{-\rho}{K_{T}}, \\
1 & \text { if } u>\frac{\rho}{K_{T}}, \\
0 & \text { if }|u| \leqslant \frac{\rho}{K_{T}} .\end{cases} \\
u_{i}^{n+1}=(1-\omega) u_{i}^{n}+\omega u_{i}^{n+\frac{1}{2}} .
\end{array}
\end{array}\right.
$$

\subsection{An academic test}

In this paragraph the algorithm is tested and validated. We have chosen a benchmark studied by the group "Validation of computer codes" of French Re- 


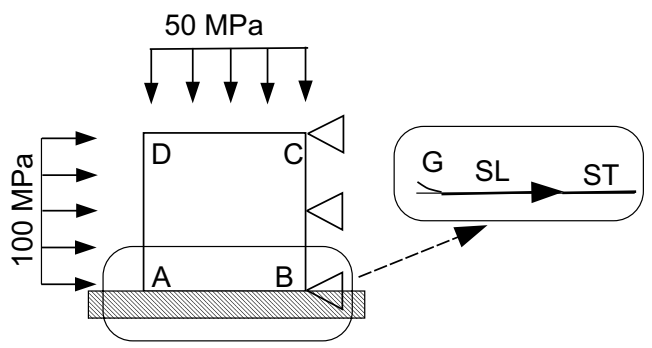

Fig. 7. The example of a long bar $(40 \mathrm{~mm} \times 40 \mathrm{~mm}): G$ corresponds to traction, SL to sliding and ST to stick zones ( $E=130 \mathrm{GPa}, v=0.2$; finite elements 3-nodes triangles).

search Groupment "Large Deformations and Damage" ([10], Fig. 7). We work in plane strains and we consider a long bar with square section $(40 \mathrm{~mm})$ with Young's modulus $E=130 \mathrm{GPa}$ and Poisson's ratio $v=0.2$. The "contact" zone (where the interface law is considered) corresponds to the part $A B$ in Fig. 7, with a friction coefficient equal to $f=1$. Boundary conditions, $u_{1}=0$ on $B C$ and $u_{1}=u_{2}=0$ at point $B$ are imposed. We apply loadings, $10 \mathrm{daN} / \mathrm{mm}^{2}$ on $A D$ and $-5 \mathrm{daN} / \mathrm{mm}^{2}$ on $C D$. In the solution of this problem we have to determine each zone of contact status.

In the finite element method, the contact zone is discretized by 32 nodes. For different values of $\bar{\mu}$ and $\bar{\lambda}$ the variations of contact status are studied (Fig. 8) and compared with the results obtained in [10] (Signorini-Coulomb laws). We observe that for large values of the coefficients we find the same results that in [10]. These coefficients correspond to the limit case (Signorini-Coulomb). The decreasing of $\bar{\mu}$ corresponds to an increasing of the stick zone (13, 15 and 32 nodes). Nevertheless, the coefficients have a strong influence on the tangential dis-

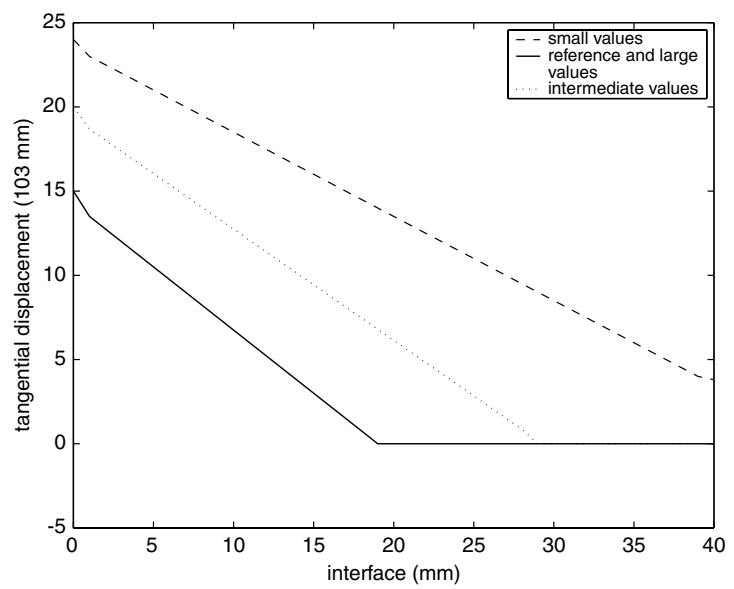

Fig. 8. Tangential displacement for various coefficient of "stiffness" $(\bar{\lambda}, \bar{\mu}):(15,5),(1510+4,510+4),(1510+8,5$ 10+8) $\mathrm{N} / \mathrm{mm}^{3}$. placements. A low value of the coefficient $K_{\mathrm{T}}$ implies an increasing of the tangential displacement. Note that the nodes are sliding without reaching the value of the usual Coulomb's sliding limit.

\section{Conclusion}

In this paper, we have modelled some non-linear thin layers by asymptotic theories. We have shown that it is possible to obtain interface laws by this kind of techniques. The main advantage of the method with respect to direct methods is a rigorous justification of the interface laws. The other advantage of the method is to find a large class of interface laws by an adapted choice of the behavior of the thin layers. In particular, we have shown how it is possible to obtain laws of Coulomb's type. One other part of our work has consisted to validate and to quantify these laws using finite elements. In the last part of the paper, we have shown how it is possible to implement these interface laws in a computational software. We have presented an efficient and robust family of algorithms which are easy to implement. We have shown that the rate of convergence of these algorithms of Gauss-Seidel's type depend only on a small number of parameters. In the future, we intend to develop other models by the same kind of techniques (other non-linear materials, large deformations, couplings), in particular for non-monotone laws [14]. The implementation of these models is necessary in order to have comparisons with experimental data.

\section{Acknowledgments}

This research was developed in part within the framework of Lagrange Laboratory, an European research group between CNRS, CNR, University of Rome "Tor Vergata", University of Montpellier II, ENPC and LCPC.

\section{References}

[1] Lebon F, Ould Khaoua A, Licht C. Numerical study of soft adhesively bonded joints in finite elasticity. Comput Mech 1998;21:134-40.

[2] C. Licht, Comportement asymptotique d'une bande dissipative mince de faible rigidité, CR Acad Sci Paris, Sér I, 1992;322:295-300.

[3] Licht C, Michaille G. A modelling of elastic adhesive bonded joints. Adv Math Sci Appl 1997;7:711-40.

[4] F. Lebon, C. Licht, F. Zaittouni, Asymptotic modelling of interfaces taking into account contact conditions. Part 1 Asymptotic expansions and numerical implementation. Part 2 Mathematical results and numerical experiments, Int J Solids Struct, to appear. 
[5] Sanchez-Hubert J, Sanchez-Palencia E. Introductions aux méthodes asymptotiques et à l'homogénéisation. Paris: Masson; 1992.

[6] Suquet P. Discontinuities and plasticity. In: Moreau JJ, Panagiotopoulos PD, editors. Nonsmooth mechanics and applications. CISM courses and lectures, vol. 302. SpringerVerlag; 1988. p. 279-340.

[7] Bayada G, Lhalouani K. Asymptotic and numerical analysis for unilateral contact problem with Coulomb's friction between an elastic body and a thin elastic soft layer. Asympt Anal 2001;25:329-62.

[8] Zaittouni F, Lebon F, Licht C. Etude théorique et numérique du comportement d'un assemblage de plaques CR Acad Sci Paris, Sér Méc 2002;330:359-64.

[9] Lemaitre J, Chaboche JL. Mécanique des matériaux solides. Paris: Dunod; 1985.

[10] Raous M, Chabrand P, Lebon F. Numerical methods for solving unilateral contact problem with friction. J Theoret Appl Mech 1988;7:111-28.
[11] Panagiotopoulos PD. Inequality problems in mechanics and applications. Convex and nonconvex energy functions. Birkhauser; 1985.

[12] Mistadikis ES, Panagiotopoulos PD. Numerical treatment of problems involving nonmonotone boundary of stressstrain laws. Comput Struct 1997;64:553-65.

[13] R. Rizzoni, Sulla torsione di cilindri di materiale elastico incomprimibile con curva tensione-deformazione non monotona. PhD Thesis. Università di Ferrara, 1999.

[14] F. Lebon, R. Rizzoni, Some considerations about soft thin layers with non-convex energies, Third Colloquium Lagrangianum, Ravello, Italy, November 6-9 2002, Springer-Verlag, in press.

[15] O. Maisonneuve, S. Pagano, P. Alart, F. Lebon, Some considerations about soft thin layers with non-convex energies. In: Argoul P, Frémond M, Nguyen QS, editors. Proceedings of IUTAM Symposium, Paris, France, 22-25 April 1997, Solids mechanics and its applications, Kluwer, 1999. p. 9-16. 